\title{
Masses of exotic calcium isotopes pin down nuclear forces
}

\author{
F. Wienholtz ${ }^{1}$, D. Beck ${ }^{2}$, K. Blaum ${ }^{3}$, Ch. Borgmann ${ }^{3}$, M. Breitenfeldt ${ }^{4}$, R. B. Cakirli ${ }^{3,5}$, S. George ${ }^{1}$, F. Herfurth ${ }^{2}$, J. D. Holt ${ }^{6,7}$, \\ M. Kowalska ${ }^{8}$, S. Kreim ${ }^{3,8}$, D. Lunney ${ }^{9}$, V. Manea ${ }^{9}$, J. Menéndez ${ }^{6,7}$, D. Neidherr ${ }^{2}$, M. Rosenbusch ${ }^{1}$, L. Schweikhard ${ }^{1}$, \\ A. Schwenk ${ }^{7,6}$, J. Simonis ${ }^{6,7}$, J. Stanja ${ }^{10}$, R. N. Wolf ${ }^{1}$ \& K. Zuber ${ }^{10}$
}

The properties of exotic nuclei on the verge of existence play a fundamental part in our understanding of nuclear interactions ${ }^{1}$. Exceedingly neutron-rich nuclei become sensitive to new aspects of nuclear forces ${ }^{2}$. Calcium, with its doubly magic isotopes ${ }^{40} \mathrm{Ca}$ and ${ }^{48} \mathrm{Ca}$, is an ideal test for nuclear shell evolution, from the valley of stability to the limits of existence. With a closed proton shell, the calcium isotopes mark the frontier for calculations with threenucleon forces from chiral effective field theory ${ }^{3-6}$. Whereas predictions for the masses of ${ }^{51} \mathrm{Ca}$ and ${ }^{52} \mathrm{Ca}$ have been validated by direct measurements ${ }^{4}$, it is an open question as to how nuclear masses evolve for heavier calcium isotopes. Here we report the mass determination of the exotic calcium isotopes ${ }^{53} \mathrm{Ca}$ and ${ }^{54} \mathrm{Ca}$, using the multi-reflection time-of-flight mass spectrometer ${ }^{7}$ of ISOLTRAP at CERN. The measured masses unambiguously establish a prominent shell closure at neutron number $N=32$, in excellent agreement with our theoretical calculations. These results increase our understanding of neutron-rich matter and pin down the subtle components of nuclear forces that are at the forefront of theoretical developments constrained by quantum chromodynamics ${ }^{8}$.

Exotic nuclei with extreme neutron-to-proton asymmetries exhibit shell structures generated by unexpected orderings of shell occupations. Their description poses enormous challenges, because most theoretical models have been developed for nuclei at the valley of stability. It is thus an open question how well they can predict new magic numbers emerging far from stability ${ }^{9-11}$. This is closely linked to our understanding of the different components of the strong force between neutrons and protons, such as the spin-orbit or tensor interactions, which modify the gaps between single-particle orbits ${ }^{12}$, and of three-body forces, which are pivotal in calculations of extreme neutron-rich systems based on nuclear forces ${ }^{2,13,14}$. The resulting magic numbers, as well as the strength of the corresponding shell closures, are critical for global predictions of the nuclear landscape ${ }^{15}$, and thus for the successful modelling of matter in astrophysical environments.

Three-body forces arise naturally in chiral effective field theory ${ }^{8}$, which provides a systematic basis for nuclear forces connected via its symmetries to the underlying theory of quarks and gluons, namely quantum chromodynamics. Owing to the consistent description in effective field theory, there are only two undetermined low-energy couplings in chiral three-nucleon forces at leading and sub-leading orders. These are constrained by the properties of light nuclei ${ }^{3} \mathrm{H}$ and ${ }^{4} \mathrm{He}$ only, so that all heavier elements are predictions in chiral effective field theory. The present frontier of three-nucleon forces is located in the calcium isotopes, where the structural evolution is dominated by valence neutrons due to the closed proton shell at atomic number $Z=20$ (refs 3,5$)$. These predictions withstood a recent challenge from direct Penning-trap mass measurements of ${ }^{51} \mathrm{Ca}$ and ${ }^{52} \mathrm{Ca}$ at TITAN/TRIUMF ${ }^{4}$, which have established a substantial change from the previous mass evaluation and leave completely open how nuclear masses evolve past ${ }^{52} \mathrm{Ca}$. This region is also very exciting because of evidence of a new magic neutron number $N=32$ from nuclear spectroscopy ${ }^{16-18}$, with a high $2^{+}$excitation energy in ${ }^{52} \mathrm{Ca}$ (refs 19, 20). These results are accompanied by successful theoretical studies based on phenomenological shell-model interactions ${ }^{21,22}$, which are similar for the excitation spectra at $N=32$ but disagree markedly in their predictions for ${ }^{54} \mathrm{Ca}$ and further away from stability.

Here we present the first mass measurements of the exotic calcium isotopes ${ }^{53} \mathrm{Ca}$ and ${ }^{54} \mathrm{Ca}$. These provide key masses for all theoretical models, and unambiguously establish a strong shell closure, in excellent agreement with the predictions including three-nucleon forces.

The mass of a nucleus provides direct access to the binding energy, the net result of all interactions between nucleons. Penning traps have proven to be the method of choice when it comes to high-precision mass determination of exotic nuclei ${ }^{23,24}$. The mass $m$ of an ion of interest with charge $q$ stored in a magnetic field $B$ is determined by comparing its cyclotron frequency $v_{\mathrm{C}}=q B /(2 \pi m)$ to that of a wellknown reference ion, $v_{\mathrm{C}, \text { Ref. }}$. The frequency ratio $r_{\mathrm{ICR}}=v_{\mathrm{C}, \mathrm{Ref}} / v_{\mathrm{C}}(\mathrm{ICR}$, ion cyclotron resonance) then yields the mass ratio directly and thus the atomic mass of the isotope.

We have made a critical step towards determining the pivotal calcium masses by introducing a new method of precision mass spectrometry for short-lived isotopes. The developments and measurements were performed with ISOLTRAP ${ }^{25}$, a high-resolution Penning-trap mass spectrometer at the ISOLDE/CERN facility. This method was used to confirm and even improve the accuracy of the recent mass measurements

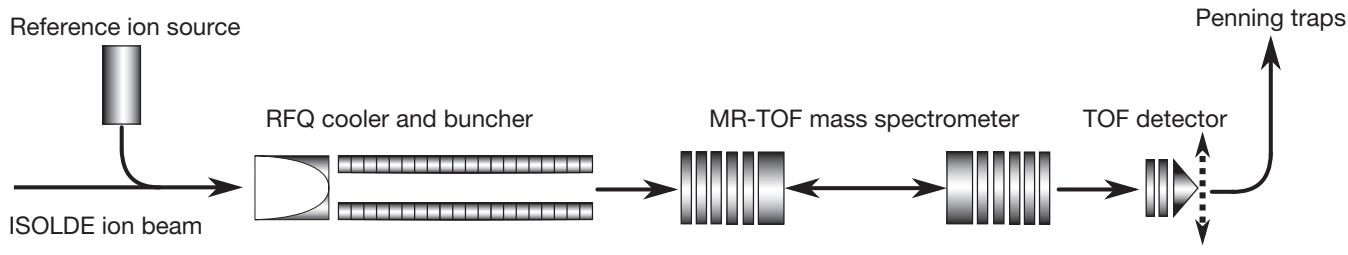

Figure 1 | Experimental set-up. Main components relevant for the ${ }^{53,54} \mathrm{Ca}$ study: incoming ISOLDE ion beam, reference ion source, radiofrequency quadrupole (RFQ) buncher, multi-reflection time-offlight (MR-TOF) mass spectrometer and (removable) time-of-flight ion detector. 
of ${ }^{51} \mathrm{Ca}$ and ${ }^{52} \mathrm{Ca}$ (ref. 4). To advance past ${ }^{52} \mathrm{Ca}$, we added a multireflection time-of-flight mass spectrometer/separator ${ }^{26}$ (MR-TOF MS, see Fig. 1) to the three other ion traps that constitute ISOLTRAP, namely a linear Paul trap and two Penning traps (the latter are not shown in Fig. 1). In the MR-TOF MS, flight paths of several kilometres are folded into table-top dimensions. This device provides not only a mass-resolving power of more than $10^{5}$, but also a mass uncertainty in the sub-parts-per-million (sub-p.p.m.) range. As typical flight times are about $10 \mathrm{~ms}$, nuclides with half-lives of the same order are accessible. Likewise, nuclei with a lower production rate can be accessed, pushing the limits currently set by Penning-trap mass spectrometry to isotopes farther away from stability.

The neutron-rich calcium isotopes were produced at the online isotope separator ISOLDE in proton-induced fission reactions of a uranium carbide target at $1.4 \mathrm{GeV}$ proton energy. The nuclides of interest were ionized by a highly selective, three-step laser-excitation scheme $^{27}$. The ions were accelerated and transported to the ISOLTRAP set-up via ISOLDE's high-resolution separator as an essentially continuous $30 \mathrm{keV}$ beam. They were captured and cooled in the radiofrequency quadrupole (RFQ) buncher and forwarded to the MR-TOF $\mathrm{MS}$ as bunches of about $60 \mathrm{~ns}$ duration. In the case of ${ }^{51} \mathrm{Ca}^{+}$and ${ }^{52} \mathrm{Ca}^{+}$, the MR-TOF MS was operated as an isobar separator, delivering the purified bunches to the Penning traps, where the mass measurements were performed by determining the cyclotron-frequency ratios as described above. Nevertheless, for ${ }^{53} \mathrm{Ca}^{+}$and ${ }^{54} \mathrm{Ca}^{+}$the Penning-trap measurements were not possible because of the low production rates and copious isobaric contamination. For example, only a few ${ }^{54} \mathrm{Ca}$ ions per minute were detected behind the MR-TOF system, accompanied by several thousand contaminating ${ }^{54} \mathrm{Cr}$ ions. The rate of delivery of ${ }^{54} \mathrm{Ca}^{+}$to the Penning traps was considerably reduced owing to the lower transport efficiency and the decay losses caused by the required extra ion trapping time.

Thus for ${ }^{53} \mathrm{Ca}^{+}$and ${ }^{54} \mathrm{Ca}^{+}$the MR-TOF device itself was employed as a mass spectrometer, where the time of flight $t$ of an ion is related to the mass-over-charge ratio $m / q$ by $t=\alpha(m / q)^{1 / 2}+\beta$. Measuring the time of flight of two well-known reference ions, here ${ }^{39} \mathrm{~K}$ and ${ }^{53 / 54} \mathrm{Cr}$ (see Fig. 2), determines the experimental parameters $\alpha$ and $\beta$. With this calibration the mass $m$ of the ions of interest, ${ }^{53} \mathrm{Ca}$ and ${ }^{54} \mathrm{Ca}$, results directly from their time of flight. This relation can be expressed by $m^{1 / 2}=C_{\mathrm{TOF}} \Delta_{\mathrm{Ref}}+\Sigma_{\mathrm{Ref}} / 2$, where $\Delta_{\mathrm{Ref}}=m_{1}{ }^{1 / 2}-m_{2}{ }^{1 / 2}$ is the difference and $\Sigma_{\text {Ref }}=m_{1}{ }^{1 / 2}+m_{2}{ }^{1 / 2}$ is the sum of the square roots of the masses of the two reference ions. $C_{\mathrm{TOF}}=\left(2 t-t_{1}-t_{2}\right) /\left[2\left(t_{1}-t_{2}\right)\right]$ comprises all measured time-of-flight values $t, t_{1}$ and $t_{2}$ of the ion of interest and the reference ions, respectively. Thus, it relates the mass $m$ of the ion of interest to the reference-ion masses $m_{1,2}$ and allows re-evaluation of the data if the value of the reference masses changes.

Our application of the MR-TOF MS method is the first for rare isotope beams. Figure 2a shows a typical time-of-flight spectrum of the mass-53 ions, which resulted from the addition of 47,000 singleshot spectra (experimental cycles) taken over a period of about $3.5 \mathrm{~h}$. The typical timescale for an 'experimental cycle', that is, the time from proton impact, after which we collect an ion ensemble, until its ejection from the MR-TOF device and detection, is of the order of $10 \mathrm{~ms}$. Figure $2 \mathrm{~b}$ shows similar spectra for mass 54 in the form of a twodimensional colour-coded intensity plot as a function of time-of-flight
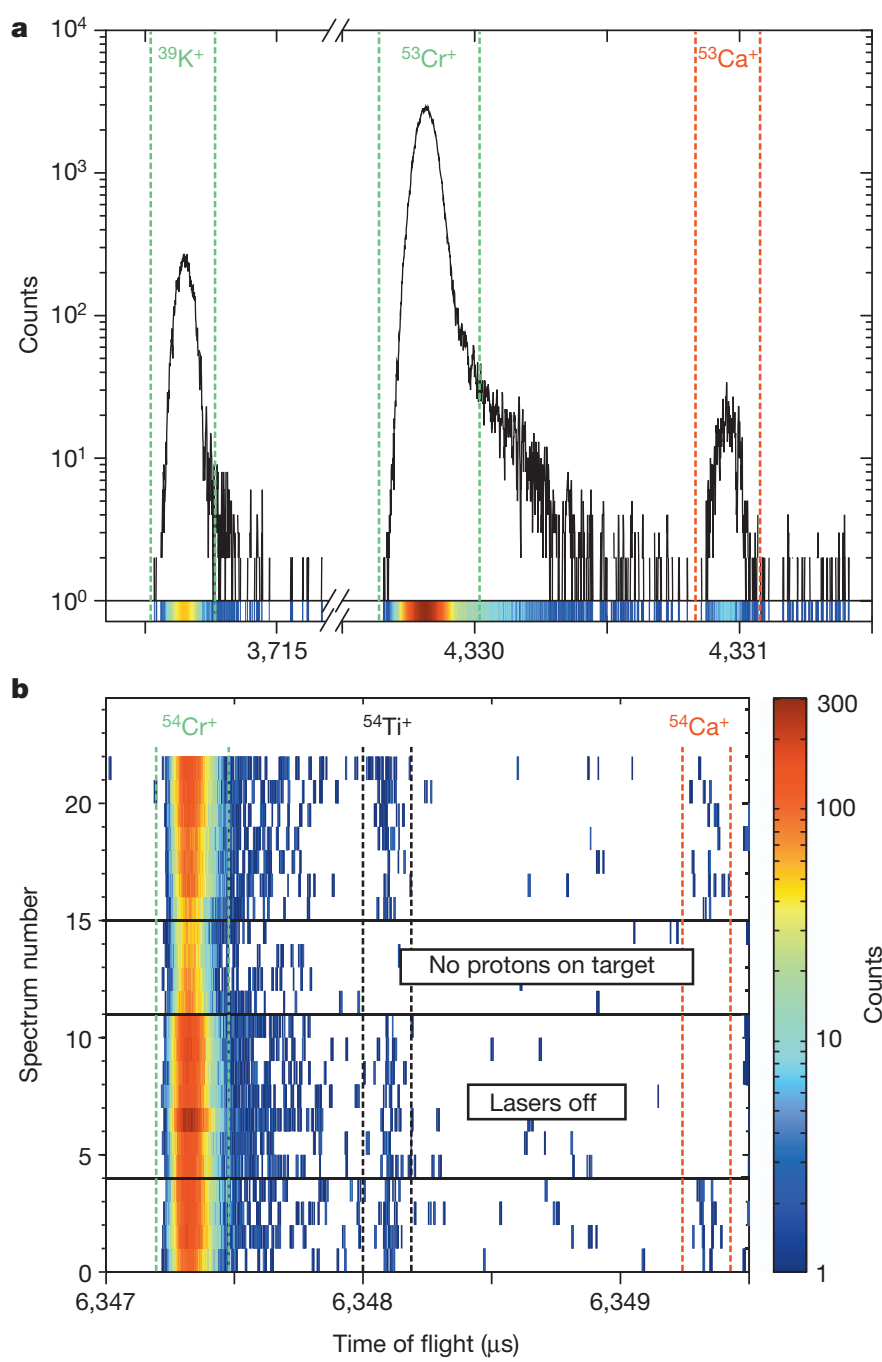

Figure $2 \mid$ Time-of-flight spectra. a, Time-of-flight spectrum of $A=53$ nuclides delivered from ISOLDE $\left({ }^{53} \mathrm{Cr}^{+},{ }^{53} \mathrm{Ca}^{+}\right)$and the reference ion ${ }^{39} \mathrm{~K}^{+}$ from the offline ion source. At bottom is the same spectrum compressed to a plot with colour-coded ion counts. b, Two-dimensional colour-coded intensity plot of time-of-flight spectra of $A=54$ nuclides. The number of ion counts (colour coded, key at right) is shown as a function of time of flight on the abscissa and as a function of the measurement time (spectrum number) on the ordinate. Intensity plots are shown for different experimental conditions (with laser ionization on and protons on target, unless indicated otherwise).

(abscissa) and spectrum number (ordinate), where each number corresponds to the accumulated data of a spectrum like that in Fig. 2a. For this particular series of measurements, the proton bombardment on the ISOLDE target was interrupted for spectra 12 to 15 to exclude the possibility that counts detected in the time region of ${ }^{54} \mathrm{Ca}^{+}$originated from any long-lived species. As expected, all short-lived species disappear from the spectrum (the production of stable ${ }^{54} \mathrm{Cr}^{+}$decreases as well). In addition, the highly selective laser ionization was switched off

Table 1 Results of the calcium mass measurements

\begin{tabular}{|c|c|c|c|c|c|c|c|}
\hline \multirow[t]{2}{*}{ Isotope } & \multirow[t]{2}{*}{$T_{1 / 2}$} & \multirow[t]{2}{*}{ Meas. type } & \multirow[t]{2}{*}{ Ref. nuclide(s) } & \multirow[t]{2}{*}{$r_{\text {ICR }}$} & \multirow[t]{2}{*}{$C_{\text {TOF }}$} & \multicolumn{2}{|c|}{ Mass excess $\left(\mathrm{keV} / \mathrm{c}^{2}\right)$} \\
\hline & & & & & & ISOLTRAP & TITAN \\
\hline${ }^{51} \mathrm{Ca}$ & $10.0(8) \mathrm{s}$ & ICR & ${ }^{39} \mathrm{~K}$ & $1.3079136760(144)$ & NA & $-36332.07(0.58)$ & $-36338.9(22.7)$ \\
\hline${ }^{52} \mathrm{Ca}$ & $4.6(3) \mathrm{s}$ & ICR & ${ }^{39} \mathrm{~K}$ & $1.3336358720(184)$ & NA & $-34266.02(0.71)$ & $-34244.6(61.0)$ \\
\hline & & MR-TOF & ${ }^{39} \mathrm{~K},{ }^{52} \mathrm{Cr}$ & NA & $0.501632110(785)$ & $-34271.7(10.2)$ & \\
\hline${ }^{53} \mathrm{Ca}$ & $461(90) \mathrm{ms}$ & MR-TOF & ${ }^{39} \mathrm{~K},{ }^{53} \mathrm{Cr}$ & NA & $0.50184761(309)$ & $-29387.8(43.3)$ & - \\
\hline${ }^{54} \mathrm{Ca}$ & $90(6) \mathrm{ms}$ & MR-TOF & ${ }^{39} \mathrm{~K},{ }^{54} \mathrm{Cr}$ & NA & $0.50210648(323)$ & $-25161.0(48.6)$ & - \\
\hline
\end{tabular}

$T_{1 / 2}$, half-life ${ }^{30}$; measurement (meas.) type (ICR, ion cyclotron resonance; MR-TOF, multi-reflection time-of-flight mass spectrometry); reference (ref.) nuclide(s) used for the calibration; $r_{\text {ICR, }}$ experimental

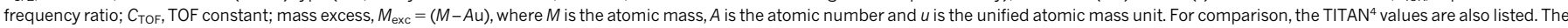
mass values of the reference nuclides are $m\left({ }^{39} \mathrm{~K}\right)=38963706.4864(49) \mu \mathrm{u}, m\left({ }^{52} \mathrm{Cr}\right)=51940506.26(63) \mu \mathrm{u}, m\left({ }^{53} \mathrm{Cr}\right)=52940648.17(62) \mu \mathrm{u}, m\left({ }^{54} \mathrm{Cr}\right)=53938879.18(61) \mu \mathrm{u}(\mathrm{ref} .28) . \mathrm{NA}, \mathrm{not}$ applicable. 


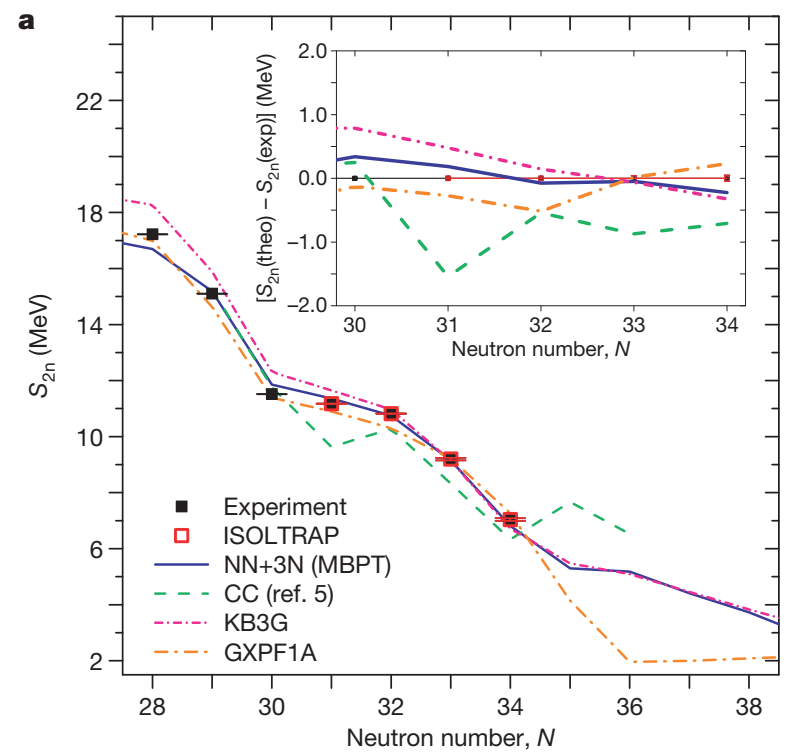

b
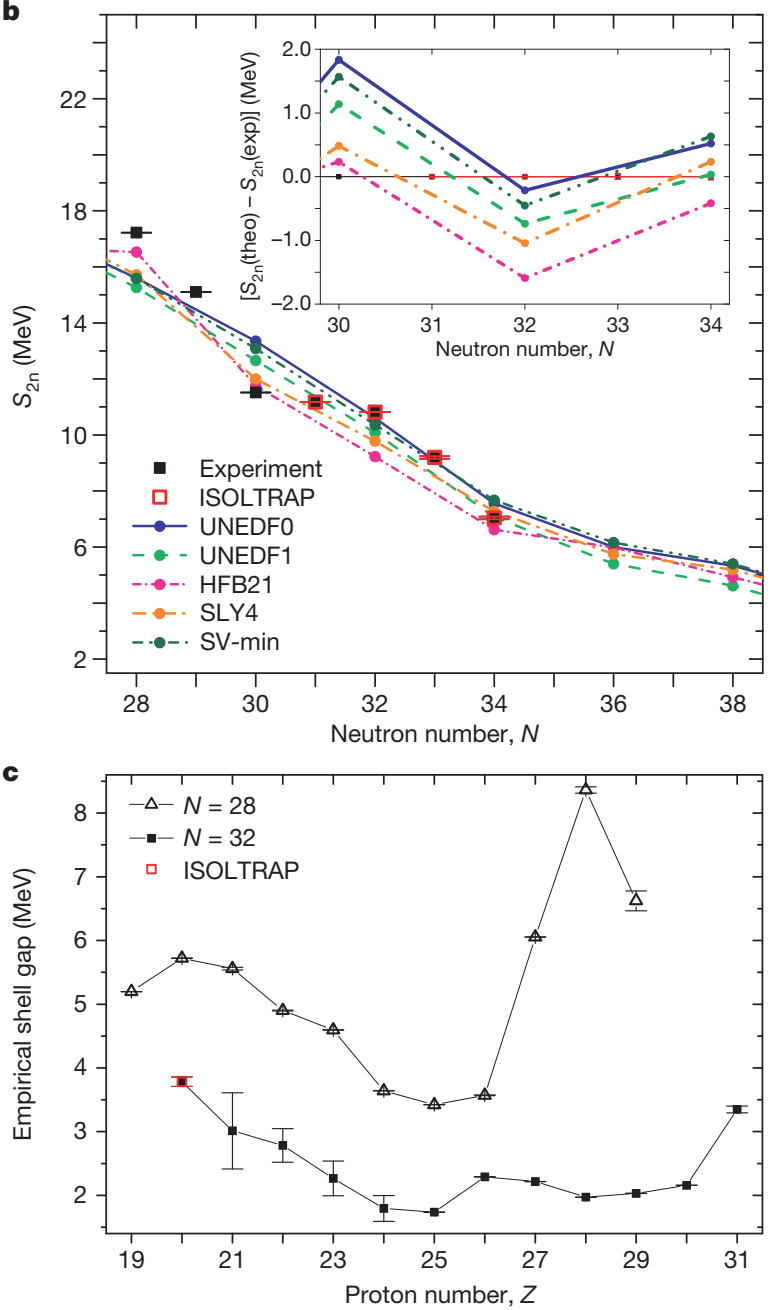

during spectra 5 to 11 , which resulted in the disappearance of the ion counts in question. This unambiguously identified these ions as ${ }^{54} \mathrm{Ca}$. Figure $2 \mathrm{~b}$ corresponds to about $90 \mathrm{~min}$ of data-taking. MR-TOF MS spectra of ${ }^{53} \mathrm{Ca}$ and ${ }^{54} \mathrm{Ca}$ were taken in total for $12.6 \mathrm{~h}$ and $18.2 \mathrm{~h}$, respectively.

Our results $\left(r_{\mathrm{ICR}}\right.$ and $\left.C_{\mathrm{TOF}}\right)$ for the exotic calcium isotopes investigated $\left({ }^{51,52} \mathrm{Ca}\right.$ and ${ }^{53,54} \mathrm{Ca}$, respectively) are summarized in Table 1 , including the resulting mass excesses. The ISOLTRAP values of ${ }^{51} \mathrm{Ca}$
Figure 3 Comparison of experimental results with theoretical predictions. a, b, Two-neutron separation energy $S_{2 n}$ (ref. 28) of the neutron-rich calcium isotopes as a function of neutron number $N$, where the new ISOLTRAP values are shown in red. In a, the ISOLTRAP masses are compared to predictions from microscopic valence-shell calculations with three-nucleon forces $(\mathrm{NN}+3 \mathrm{~N})$ based on chiral effective field theory (solid line, MBPT) and large-space coupledcluster calculations including three-nucleon forces as density-dependent twobody interactions (dashed line, $\mathrm{CC})^{5}$. For comparison, we also show the results of the phenomenological shell-model interactions $\mathrm{KB}_{3} \mathrm{G}^{21}$ and $\mathrm{GXPF}_{1} \mathrm{~A}^{22}$. In b, the ISOLTRAP masses are compared to state-of-the-art nuclear densityfunctional-theory prediction $s^{15,29}$. Insets in $\mathbf{a}$ and $\mathbf{b}$ show the difference between the theoretical predictions and experiment. c, Empirical two-neutron shell gap as a function of proton number $Z$ for $N=28$ and $N=32$. Error bars, \pm 1 s.d.

and ${ }^{52} \mathrm{Ca}$ determined with the Penning trap agree well with the recent measurements by TITAN ${ }^{4}$. The uncertainties were reduced by factors of 40 and 80 , respectively, owing to longer excitation times $(600 \mathrm{~ms}$ in the case of ISOLTRAP as compared to $80 \mathrm{~ms}$ in the case of TITAN), higher cyclotron frequencies and higher calcium ion yields. The masses of ${ }^{53,54} \mathrm{Ca}$ determined by the MR-TOF MS have been experimentally addressed for the first time. As a consistency check, the ${ }^{52} \mathrm{Ca}$ mass was also measured by the new MR-TOF method, and the mass excess is in full agreement with both Penning-trap results (Table 1). Furthermore, a second cross-check measurement in the vicinity of the newly measured masses was performed. The mass excess of the stable isotope ${ }^{58} \mathrm{Fe}$ was determined with the stable reference isotopes ${ }^{58} \mathrm{Ni}$ and ${ }^{85} \mathrm{Rb}$. The measurement resulted in a mass excess of $-62,168.0(47.0) \mathrm{keV} / \mathrm{c}^{2}$, where the statistical uncertainty is given in parentheses. With a deviation of $13.5 \mathrm{keV} / \mathrm{c}^{2}$ from the literature value $\mathrm{e}^{28}$, it agrees well within its statistical uncertainty. The uncertainties in the MR-TOF method quoted in Table 1 for ${ }^{53} \mathrm{Ca}$ and ${ }^{54} \mathrm{Ca}$ denote the statistical standard deviation. For the cross-checks, the MR-TOF method has thus been employed to measure the mass of a slightly lighter isotope and a slightly heavier isotope, ${ }^{52} \mathrm{Ca}$ and ${ }^{58} \mathrm{Fe}$, respectively. The deviations from the Penning-trap measurement and the literature value, respectively, are taken as estimates of the relative systematic uncertainty, which lies in the low $10^{-7}$ range. Additional cross-check measurements to determine the systematic uncertainty have been performed over a wide mass range and will be detailed elsewhere. The precision and fast measurement cycle of the MR-TOF method makes this a promising approach for the mass spectrometry of isotopes with lower yield and shorter half-life than currently accessible.

The binding energies encode information about the ordering of shell occupation, and thus are essential in the quest for shell closures in exotic regions of the nuclear chart. Our high-precision data can be used to provide a critical benchmark for the behaviour far from stability, namely, the two-neutron separation energy $S_{2 \mathrm{n}}=B(Z, N)-B(Z, N-2)$, where $B(Z, N)$ is the binding energy (defined as positive) of a nucleus with $Z$ protons and $N$ neutrons. The $S_{2 n}$ values are a preferred probe of the evolution of nuclear structure with neutron number, and can be used to challenge model predictions, as shown in Fig. 3. The pronounced decrease in $S_{2 n}$ revealed by the new ${ }^{53} \mathrm{Ca}$ and ${ }^{54} \mathrm{Ca}$ ISOLTRAP masses is similar to the decrease beyond the doubly magic ${ }^{48} \mathrm{Ca}$. In general, correlations induced by deformation could also cause such a reduction in $S_{2 \mathrm{n}}$, but in the calcium isotopes studied here deformation is expected to have no role ${ }^{29}$. Therefore, our new data unambiguously establish a prominent shell closure at $N=32$. The strength of this shell closure can be evaluated from the two-neutron shell gap, that is, the two-neutron separation energy difference $S_{2 \mathrm{n}}(Z, N)-S_{2 \mathrm{n}}(Z, N+2)$. Figure $3 \mathrm{c}$ shows a two-neutron shell gap for ${ }^{52} \mathrm{Ca}$ of almost $4 \mathrm{MeV}$, where the rise towards ${ }^{52} \mathrm{Ca}$ at $N=32$ is as steep as that towards ${ }^{48} \mathrm{Ca}$ at $N=28$. The peaks at $N=Z$ in Fig. $3 c$ are due to the additional correlation energy for symmetric $N=Z$ nuclei, known as Wigner energy.

Calcium marks the heaviest chain of isotopes studied with threenucleon forces based on chiral effective field theory ${ }^{3-6}$. Figure 3 a shows the predictions of our microscopic calculations with three-nucleon forces (that is, ' $\mathrm{NN}+3 \mathrm{~N}$ ') using many-body perturbation theory 
(MBPT) for the valence-neutron interactions ${ }^{3,4}$; it also shows predictions of large-scale coupled-cluster ('CC') calculations including the continuum and three-nucleon forces as density-dependent two-body interactions ${ }^{5}$. Confronted with the $S_{2 n}$ values obtained from the new masses of ${ }^{53} \mathrm{Ca}$ and ${ }^{54} \mathrm{Ca}$, we find an excellent agreement with the predictions. We have also calculated perturbatively the effect of residual three-valence-neutron forces. This provides only a very small repulsive contribution, lowering $S_{2 n}$ by about $50 \mathrm{keV}$ to $250 \mathrm{keV}$ from ${ }^{51} \mathrm{Ca}$ to ${ }^{54} \mathrm{Ca}$ in the MBPT framework. The agreement of the $\mathrm{NN}+3 \mathrm{~N}$ calculations with the new ISOLTRAP masses is remarkable, because their parameters are fitted only to the properties of few-nucleon systems while their level of accuracy here is similar to the phenomenological shell-model interactions $\mathrm{KB} \mathrm{G}^{21}$ and $\mathrm{GXPF} 1 \mathrm{~A}^{22}$, which are adjusted to the medium-mass region. The CC calculations predict a $N=32$ shell gap very close to our measurements; however, the oscillations in $S_{2 n}$ for odd neutron numbers on either side of the shell closure disagree with experiment.

In Fig. 3b we compare the new ISOLTRAP masses to state-of-theart nuclear density-functional-theory (DFT) predictions ${ }^{15,29}$, which have recently provided global predictions for nuclear driplines ${ }^{15}$. (The dripline marks the limit of existence where nuclei cease to be bound.) This shows that modern DFT calculations can reproduce the masses of ${ }^{52-54} \mathrm{Ca}$. In particular, the UNEDF0 and SV-min functionals are in very good agreement. However, the DFT calculations predict an almost linear progression of the two-neutron separation energy that does not lead to the experimentally observed decrease in $S_{2 n}$ at ${ }^{48} \mathrm{Ca}$ and at ${ }^{52} \mathrm{Ca}$, established by the new masses of ${ }^{53} \mathrm{Ca}$ and ${ }^{54} \mathrm{Ca}$. In the $\mathrm{NN}+3 \mathrm{~N}$ calculations, the neutron dripline is obtained at ${ }^{62} \mathrm{Ca}$, but owing to the very flat behaviour of the binding energies past ${ }^{60} \mathrm{Ca}$ (refs 3,5 , $15,29)$, the limit of calcium isotopes is very difficult to predict theoretically. In addition, the effects of the continuum, not included in the MBPT calculations, will be decisive for nuclides close to the dripline $e^{5,29}$. Note that the calcium isotopes studied here are still well bound, with 4.2 MeV separation energy in ${ }^{54} \mathrm{Ca}$, so that the effects of the continuum on the ground-state energies are small (of the order of $100 \mathrm{keV})^{5}$, comparable to the small effects of residual three-nucleon forces.

The present results based on precision mass measurements with a multi-reflection time-of-flight method reinforce the suggestion that pronounced structural effects are important in exotic nuclei and that shell effects do not smear out far from stability. Our results provide useful information for all theoretical models, and they show that a description of extreme neutron-rich nuclei can be closely connected to a deeper understanding of nuclear forces. Chiral effective field theory provides this connection and an exciting framework for exploring neutron-rich nuclei. The measurements of the ${ }^{53} \mathrm{Ca}$ and ${ }^{54} \mathrm{Ca}$ isotopes, accessed in this work, present anchor points to pin down nuclear forces. Finally, we note that the advantages of the MR-TOF method as compared to Penning-trap mass spectrometry will also be important for new experimental facilities, which will provide even more exotic ion beams. The present and future developments of low-energy beams at facilities for the study of exotic nuclides such as ARIEL, CARIBU, FAIR, FRIB, HIE-ISOLDE, RIBF and SPIRAL 2 will considerably extend the available range of rare isotopes towards the nuclear driplines. The minute production rates of isotopes with half-lives in the millisecond range and substantial isobaric contamination pose experimental challenges that are barely met by Penning traps now, but can be overcome with the MR-TOF method.

\section{Received 19 December 2012; accepted 23 April 2013.}

1. Baumann, T., Spyrou, A. \& Thoennessen, M. Nuclear structure experiments along the neutron drip line. Rep. Prog. Phys. 75, 036301 (2012).
2. Hammer, H.-W., Nogga, A. \& Schwenk, A. Three-body forces: from cold atoms to nuclei. Rev. Mod. Phys. 85, 197-217 (2013).

3. Holt, J. D. et al. Three-body forces and shell structure in calcium isotopes. J. Phys. G 39, 085111 (2012)

4. Gallant, A. T.etal. New precision mass measurements of neutron-rich calcium and potassium isotopes and three-nucleon forces. Phys. Rev. Lett. 109, 032506 (2012).

5. Hagen, G. et al. Evolution of shell structure in neutron-rich calcium isotopes. Phys. Rev. Lett. 109, 032502 (2012).

6. Roth, R. et al. Medium-mass nuclei with normal-ordered chiral NN+3N interactions. Phys. Rev. Lett. 109, 052501 (2012).

7. Wollnik, H. \& Przewloka, M. Time-of-flight mass spectrometers with multiply reflected ion trajectories. Int. J. Mass Spectrom. Ion Process. 96, 267-274 (1990).

8. Epelbaum, E., Hammer, H.-W. \& Meißner, U.-G. Modern theory of nuclear forces. Rev. Mod. Phys. 81, 1773-1825 (2009).

9. Warner, D. Not-so-magic numbers. Nature 430, 517-519 (2004).

10. Sorlin, O.\& Porquet, M.-G. Nuclear magic numbers: new features far from stability. Prog. Part. Nucl. Phys. 61, 602-673 (2008)

11. Janssens, R. V. F. Unexpected doubly magic nucleus. Nature 459, 1069-1070 (2009).

12. Otsuka, T. et al. Magic numbers in exotic nuclei and spin-isospin properties of the NN interaction. Phys. Rev. Lett. 87, 082502 (2001).

13. Otsuka, T. et al. Three-body forces and the limit of oxygen isotopes. Phys. Rev. Lett. 105, 032501 (2010)

14. Hagen, G. etal. Continuum effects and three-nucleon forces in neutron-rich oxygen isotopes. Phys. Rev. Lett. 108, 242501 (2012).

15. Erler, J. et al. The limits of the nuclear landscape. Nature 486, 509-512 (2012).

16. Janssens, R. V. F. et al. Structure of ${ }^{52,54} \mathrm{Ti}$ and shell closures in neutron-rich nuclei above ${ }^{48}$ Ca. Phys. Lett. B 546, 55-62 (2002).

17. Mantica, P. F. et al. $\beta$ decay of neutron-rich ${ }^{53-56}$ Ca. Phys. Rev. C 77, 014313 (2008).

18. Crawford, H. L. et al. $\beta$ decay and isomeric properties of neutron-rich $\mathrm{Ca}$ and $\mathrm{Sc}$ isotopes. Phys. Rev. C 82, 014311 (2010).

19. Huck, A. etal. Beta decay of the new isotopes ${ }^{52} \mathrm{~K},{ }^{52} \mathrm{Ca}$, and ${ }^{52} \mathrm{Sc}$; a test of the shell model far from stability. Phys. Rev. C 31, 2226-2237 (1985)

20. Gade, A. et al. Cross-shell excitation in two-proton knockout: structure of ${ }^{52} \mathrm{Ca}$. Phys. Rev. C 74, 021302(R) (2006).

21. Poves, A. et al. Shell model study of the isobaric chains $A=50, A=51$ and $A=52$. Nucl. Phys. A 694, 157-198 (2001).

22. Honma, M. et al. New effective interaction for pf-shell nuclei and its implications for the stability of the $\mathrm{N}=\mathrm{Z}=28$ closed core. Phys. Rev. C 69, 034335 (2004).

23. Blaum, K. High-accuracy mass spectrometry with stored ions. Phys. Rep. $\mathbf{4 2 5}$, $1-78$ (2006).

24. Schweikhard, L. \& Bollen, G. (eds) Special issue on ultra-accurate mass spectrometry and related topics. Int. J. Mass Spectrom. 251, 85-312 (2006).

25. Mukherjee, M. et al. ISOLTRAP: an on-line Penning trap for mass spectrometry on short-lived nuclides. Eur. Phys. J. A 35, 1-29 (2008).

26. Wolf, R. N. et al. On-line separation of short-lived nuclei by a multi-reflection timeof-flight device. Nucl. Instrum. Methods A 686, 82-90 (2012).

27. Fedosseev, V. N. et al. Upgrade of the resonance ionization laser ion source at ISOLDE on-line isotope separation facility: new lasers and new ion beams. Rev. Sci. Instrum. 83, 02 A903 (2012).

28. Wang, M. et al. The AME2012 atomic mass evaluation. Chinese Phys. C 36, 1603-2014 (2012)

29. Forssén, C. et al. Living on the edge of stability, the limits of the nuclear landscape. Phys. Scr. T 152, 014022 (2013).

30. Audi, G. et al. The NUBASE2012 evaluation of nuclear properties. Chinese Phys. C 36, 1157-1286 (2012).

Acknowledgements This work was supported by the BMBF (contracts 06GF9102, 05P12HGCl1, 05P12HGFNE, 06DA70471,06DD9054), the DFG (grants SFB 634 and GE2183/2-1), the ERC (grant 307986 STRONGINT), the EU through ENSAR (grant 262010), the Helmholtz Alliance HA216/EMMI, the French IN2P3, the ISOLDE Collaboration and the Max-Planck Society. Computations were performed at the Jülich Supercomputing Center.

Author Contributions D.B., Ch.B., R.B.C. S.K. D.L., V.M., D.N., M.R., J. Stanja, F.W. and R.N.W. performed the experiment. V.M. and F.W. performed the data analysis. J.D.H., J.M.., A.S. and J. Simonis performed the NN+3N (MBPT) calculations. K.B., S.K., D.L., A.S., L.S. and F.W. prepared the manuscript. All authors discussed the results and contributed to the manuscript at all stages.

Author Information Reprints and permissions information is available at www.nature.com/reprints. The authors declare no competing financial interests. Readers are welcome to comment on the online version of the paper. Correspondence and requests for materials should be addressed to F.W. (wienholtz@physik.uni-greifswald.de). 
ERRATUM

doi:10.1038/nature 12431

\section{Erratum: Masses of exotic calcium isotopes pin down nuclear forces}

F. Wienholtz, D. Beck, K. Blaum, Ch. Borgmann, M. Breitenfeldt, R. B. Cakirli, S. George, F. Herfurth, J. D. Holt, M. Kowalska, S. Kreim, D. Lunney, V. Manea, J. Menéndez, D. Neidherr, M. Rosenbusch, L. Schweikhard, A. Schwenk, J. Simonis, J. Stanja, R. N. Wolf \& K. Zuber

Nature 498, 346-349 (2013); doi:10.1038/nature12226

In Fig. $3 \mathrm{~b}$ of this Letter, the $y$ axis numbering should be identical to that of Fig. 3a, and start at $2 \mathrm{MeV}$ and go up to $25 \mathrm{MeV}$. Instead, the numbering was inadvertently shifted upwards, which made the data shown in Fig. $3 \mathrm{~b}$ appear to have higher values by $2 \mathrm{MeV}$ with respect to their actual ones. Figure 3 has been corrected in the HTML and PDF of the original manuscript. 